\title{
Morteza Djamali, Matthew D. Jones, Jérémie Migliore, Silvia Balatti, Marianela Fader, Daniel Contreras, Sébastien Gondet, Zahra Hosseini, Hamid Lahijani, Abdolmajid Naderi, Lyudmila S. Shumilovskikh, Margareta Tengberg, Lloyd Weeks. "Olive cultivation in the heart of the Persian Achaemenid Empire: new insights into agricultural practices and environmental changes reflected in a late Holocene pollen record from Lake Parishan, SW Iran »
}

Rémy Boucharlat

\section{(QpenEdition Journals}

Édition électronique

URL : http://journals.openedition.org/abstractairanica/46689

DOI : $10.4000 / a b s t r a c t a i r a n i c a .46689$

ISBN : 1961-960X

ISSN : 1961-960X

Éditeur :

CNRS (UMR 7528 Mondes iraniens et indiens), Éditions de l'IFRI

\section{Référence électronique}

Rémy Boucharlat, « Morteza Djamali, Matthew D. Jones, Jérémie Migliore, Silvia Balatti, Marianela Fader, Daniel Contreras, Sébastien Gondet, Zahra Hosseini, Hamid Lahijani, Abdolmajid Naderi, Lyudmila S. Shumilovskikh, Margareta Tengberg, Lloyd Weeks. « Olive cultivation in the heart of the Persian Achaemenid Empire: new insights into agricultural practices and environmental changes reflected in a late Holocene pollen record from Lake Parishan, SW Iran » », Abstracta Iranica [En ligne], Volume 37-38-39 | 2018, document 85, mis en ligne le 30 décembre 2018, consulté le 02 octobre 2020. URL : http://journals.openedition.org/abstractairanica/46689; DOI : https://doi.org/10.4000/ abstractairanica.46689 
Morteza Djamali, Matthew D. Jones, Jérémie Migliore, Silvia Balatti, Marianela Fader, Daniel Contreras, Sébastien Gondet, Zahra Hosseini, Hamid Lahijani, Abdolmajid Naderi, Lyudmila S. Shumilovskikh, Margareta Tengberg, Lloyd Weeks. " Olive cultivation in the heart of the Persian Achaemenid Empire: new insights into agricultural practices and environmental changes reflected in a late Holocene pollen record from Lake Parishan,

\section{SW Iran »}

Rémy Boucharlat 


\section{RÉFÉRENCE}

Morteza Djamali, Matthew D. Jones, Jérémie Migliore, Silvia Balatti, Marianela Fader, Daniel Contreras, Sébastien Gondet, Zahra Hosseini, Hamid Lahijani, Abdolmajid Naderi, Lyudmila S. Shumilovskikh, Margareta Tengberg, Lloyd Weeks. «Olive cultivation in the heart of the Persian Achaemenid Empire: new insights into agricultural practices and environmental changes reflected in a late Holocene pollen record from Lake Parishan, SW Iran ", Vegetation History and Archaeobotany, vol. 25/3, 2015-2016, p. 255-269.

1 La période des grands empires, des Achéménides aux Sassanides, est marquée par une remarquable expansion de l'arboriculture, ce qu'un article antérieur de certains des auteurs de cet article avait déjà montré pour le platane à partir de la fin du IIe mill. av.n.è., et plus encore, selon les auteurs, sous l'impulsion de l'empire achéménide (analyses de pollens dans le lac Maharlou au SE de Persépolis, cf. Morteza Djamali et al., "Notes on Arboricultural and Agricultural Practices in Ancient Iran based on New Pollen Evidence", Paléorient 36, 2, 2010, p. 175-18). Cette fois, ce sont les résultats d'analyses des restes polliniques du lac Parishan, zone de marais près de Kaezerun qui montrent que l'arboriculture était l'activité agricole majeure des Achéménides aux Parthes, en particulier la culture de l'olivier, très importante dans le secteur du lac, ainsi que la vigne et le noyer. À cette époque le niveau du lac était supérieur à l'actuel, favorisant sans doute la prospérité socio-économique. L'absence de mention de l'époque sassanide dans les résultats dans cette histoire de l'évolution est surprenante, car elle est, selon des données textuelles et archéologiques, une période d'intense activité agricole. Cette contribution et d'autres depuis quelques années rendent compte de la prise en compte des périodes archéologiques « récentes » dans les études paléoenvironnementales.

\section{AUTEURS}

\section{RÉMY BOUCHARLAT}

UMR 5133 CNRS-Université de Lyon 